\title{
COLLAPSED SYMMETRY MODEL AND ITS DECOMPOSITION FOR MULTI-WAY TABLES WITH ORDERED CATEGORIES
}

\author{
Kouji Tahata*, Akira Takazawa* and Sadao Tomizawa*
}

For $r \times r$ tables with ordered categories, Tomizawa (1995) considered the collapsed symmetry model. This model indicates the structure of symmetry for the $r-1$ ways of collapsing the $r \times r$ table into a $2 \times 2$ table by choosing cut points after the $u$-th row and after the $u$-th column for $u=1, \ldots, r-1$. This paper proposes a collapsed symmetry $(C)$ model for multi-way tables with ordered categories. The proposed model is an extension of the complete symmetry model and a special case of the marginal homogeneity $(M)$ model. Also for multi-way tables, this paper proposes the collapsed quasi-symmetry $(C Q S)$ model which is an extension of the $C$ model, and gives a theorem that the $C$ model holds if and only if both the $C Q S$ and $M$ models hold. An example is given.

Key words and phrases: Collapsed quasi-symmetry, collapsed symmetry, decomposition, marginal homogeneity, multi-way tables, ordered category, quasi-symmetry, symmetry.

\section{Introduction}

For an $r \times r$ square contingency table with the same ordinal row and column classifications, let $p_{i j}$ denote the probability that an observation will fall in the $i$-th row and $j$-th column of the table $(i=1, \ldots, r ; j=1, \ldots, r)$ and let $X_{1}$ and $X_{2}$ denote the row and column variables, respectively. The symmetry model is defined by

$$
p_{i j}=p_{j i} \quad(i=1, \ldots, r ; j=1, \ldots, r) \text {; }
$$

see, for example, Bowker (1948) and Bishop et al. (1975, p. 282). This model indicates that the probability that an observation will fall in row category $i$ and column category $j(\neq i)$ is equal to the probability that the observation falls in row category $j$ and column category $i$.

Caussinus (1965) considered the quasi-symmetry model defined by

$$
p_{i j}=\mu \alpha_{i} \beta_{j} \psi_{i j} \quad(i=1, \ldots, r ; j=1, \ldots, r),
$$

where $\psi_{i j}=\psi_{j i}$. A special case of this model obtained by putting $\left\{\alpha_{i}=\beta_{i}\right\}$ is the symmetry model.

The marginal homogeneity model is defined by

$$
p_{i .}=p_{\cdot i} \quad(i=1, \ldots, r)
$$

Received July 20, 2007. Revised January 16, 2008. Accepted January 18, 2008.

*Department of Information Sciences, Faculty of Science and Technology, Tokyo University of Science, Noda City, Chiba 278-8510, Japan. Email: kouji_tahata@is.noda.tus.ac.jp, tomizawa@is.noda.tus.ac.jp 
where $p_{i} .=\sum_{s=1}^{r} p_{i s}$ and $p_{\cdot i}=\sum_{t=1}^{r} p_{t i}$ (Stuart (1955)). This model indicates that the row marginal distribution is identical to the column marginal distribution. Note that Caussinus (1965) gave the theorem that the symmetry model holds if and only if both the quasi-symmetry and marginal homogeneity models hold.

Consider the $r-1$ ways of collapsing the $r \times r$ table into a $2 \times 2$ table by choosing cut points after the $u$-th row and after the $u$-th column for $u=1, \ldots, r-1$ (see Table 1). Tomizawa $(1993,1995)$ described the collapsed symmetry (denoted by $C^{2}$ ) model, defined by

$$
G_{1(u)}=G_{2(u)} \quad(u=1, \ldots, r-1),
$$

where

$$
G_{1(u)}=\sum_{s=1}^{u} \sum_{t=u+1}^{r} p_{s t}=\mathrm{P}\left(X_{1} \leq u, X_{2}>u\right)
$$

and

$$
G_{2(u)}=\sum_{s=u+1}^{r} \sum_{t=1}^{u} p_{s t}=\mathrm{P}\left(X_{1}>u, X_{2} \leq u\right) .
$$

See also McCullagh (1978), Agresti (1984, p. 205), Tomizawa et al. (2001, 2006, 2007), Miyamoto et al. (2004), and Tomizawa and Miyamoto (2007). This model states that for $u=1, \ldots, r-1$, the cumulative probability that an observation will fall in row category $u$ or below, and in column category $u+1$ or above, is

Table 1. Illustration of (a) the $4 \times 4$ table of probabilities, and (b) the collapsed $2 \times 2$ tables.

(a) The probabilities

\begin{tabular}{cccccc}
\hline & \multicolumn{5}{c}{$X_{2}$} \\
\cline { 2 - 5 }$X_{1}$ & $(1)$ & $(2)$ & $(3)$ & $(4)$ & Total \\
\hline$(1)$ & $p_{11}$ & $p_{12}$ & $p_{13}$ & $p_{14}$ & $p_{1}$. \\
$(2)$ & $p_{21}$ & $p_{22}$ & $p_{23}$ & $p_{24}$ & $p_{2 .}$ \\
$(3)$ & $p_{31}$ & $p_{32}$ & $p_{33}$ & $p_{34}$ & $p_{3 .}$ \\
$(4)$ & $p_{41}$ & $p_{42}$ & $p_{43}$ & $p_{44}$ & $p_{4}$. \\
\hline Total & $p_{\cdot 1}$ & $p_{\cdot 2}$ & $p_{\cdot 3}$ & $p_{\cdot 4}$ & 1 \\
\hline
\end{tabular}

(b) The $u$-th $(u=1,2,3)$ collapsed $2 \times 2$ table

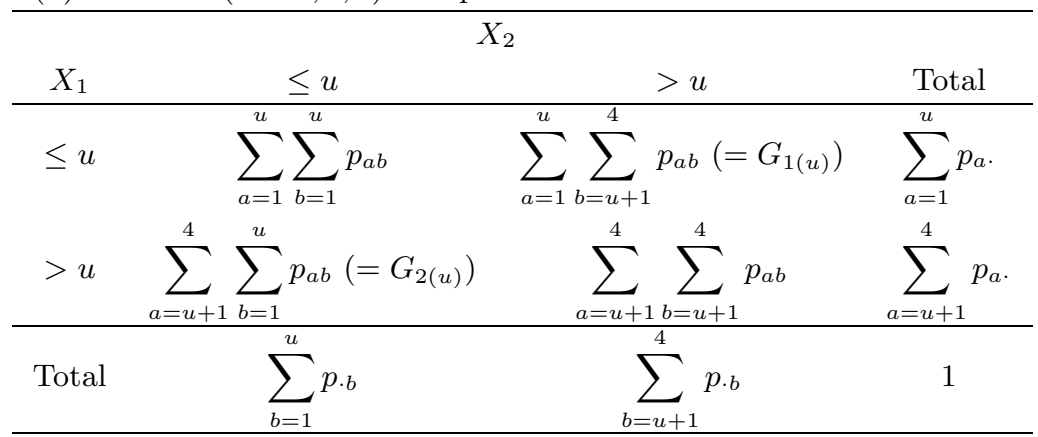


equal to the cumulative probability that the observation falls in column category $u$ or below, and in row category $u+1$ or above. This indicates that there is a structure of symmetry in each collapsed $2 \times 2$ table. We note that the collapsed symmetry model is equivalent to the marginal homogeneity model for the $r \times r$ table.

Next consider an $r^{T}$ contingency table $(T \geq 2)$. Let $p_{i_{1} \ldots i_{T}}$ denote the probability that an observation will fall in the $\left(i_{1}, \ldots, i_{T}\right)$-th cell of the table. The complete symmetry $\left(S^{T}\right)$ model is defined by

$$
p_{i_{1} \ldots i_{T}}=p_{j_{1} \ldots j_{T}},
$$

for any permutation $\left(j_{1}, \ldots, j_{T}\right)$ of $\left(i_{1}, \ldots, i_{T}\right)$ with $i_{k}=1, \ldots, r ; k=1, \ldots, T$ (Agresti (2002), p. 440). For instance, in a three-way table, $p_{i j k}=p_{i k j}=p_{j i k}=$ $p_{j k i}=p_{k i j}=p_{k j i}$ (Bishop et al. (1975), p. 301).

Bishop et al. (1975, p. 303) defined the quasi-symmetry model for three-way tables (i.e., for $T=3$ ), and Bhapkar and Darroch (1990) defined the quasisymmetry $\left(Q S^{T}\right)$ model for multi-way tables, which may be expressed as

$$
p_{i_{1} \ldots i_{T}}=\mu \alpha_{1\left(i_{1}\right)} \alpha_{2\left(i_{2}\right)} \cdots \alpha_{T\left(i_{T}\right)} \psi_{i_{1} \ldots i_{T}},
$$

where $\psi_{i_{1} \ldots i_{T}}=\psi_{j_{1} \ldots j_{T}}$ and $\left(j_{1}, \ldots, j_{T}\right)$ is any permutation of $\left(i_{1}, \ldots, i_{T}\right)$ with $i_{k}=1, \ldots, r ; k=1, \ldots, T$. For the details of this model, see also Agresti (2002, p. 440), Tomizawa and Tahata (2007), and Yamamoto et al. (2007).

Let $X_{k}(k=1, \ldots, T)$ denote the $k$-th variable. The marginal homogeneity $\left(M^{T}\right)$ model is defined by

$$
p_{i}^{(1)}=\cdots=p_{i}^{(T)} \quad(i=1, \ldots, r),
$$

where

$$
p_{i}^{(k)}=\mathrm{P}\left(X_{k}=i\right) \quad(k=1, \ldots, T) .
$$

For instance, in a three-way table, $p_{i . .}=p_{\cdot i .}=p_{. \cdot i}$ where $p_{i . .}=\sum_{s=1}^{r} \sum_{t=1}^{r} p_{i s t}$, $p_{\cdot i \cdot}=\sum_{s=1}^{r} \sum_{t=1}^{r} p_{s i t}$ and $p_{. \cdot i}=\sum_{s=1}^{r} \sum_{t=1}^{r} p_{s t i}$. Note that Bhapkar and Darroch (1990) gave the theorem that for multi-way tables, the $S^{T}$ model holds if and only if both the $Q S^{T}$ and $M^{T}$ models hold (see also Agresti (2002, p. 440), Tomizawa and Tahata (2007), Yamamoto et al. (2007)).

The $S^{T}, Q S^{T}$ and $M^{T}$ models are invariant under the arbitrarily same permutations of categories for all variables. Thus, it is suitable to apply these models for analyzing the multi-way tables with nominal categories. We are interested in the models that indicate the structure of symmetry for multi-way tables with ordered categories; concretely, in (1) extending the collapsed symmetry model to multi-way tables, in (2) proposing the collapsed quasi-symmetry model for multiway tables, and in (3) decomposing the collapsed symmetry model for multi-way tables.

The purpose of this paper is to propose two new models for multi-way tables with ordered categories. Subsection 2.1 proposes the collapsed symmetry model and the collapsed quasi-symmetry model for three-way contingency tables, and 
gives a decomposition of the collapsed symmetry model. Subsection 2.2 extends them to multi-way tables. The collapsed symmetry model is an extension of the $S^{T}$ model and a special case of the $M^{T}$ model for $T \geq 3$. The collapsed quasi-symmetry model is an extension of the collapsed symmetry model, and it is different from the quasi-symmetry model. Section 3 gives an example.

\section{Collapsed symmetry models}

\subsection{Case of three-way tables}

Consider an $r \times r \times r$ contingency table with ordered categories. Let $p_{i j k}$ denote the probability that an observation will fall in the $(i, j, k)$-th cell of the table for $1 \leq i, j, k \leq r$. Denote the $k$-th variable by $X_{k}(k=1,2,3)$.

For $u=1, \ldots, r-1$, let

$$
\begin{aligned}
& G_{1(u)}=\mathrm{P}\left(X_{1} \leq u, X_{2}>u, X_{3}>u\right), \\
& G_{2(u)}=\mathrm{P}\left(X_{1}>u, X_{2} \leq u, X_{3}>u\right), \\
& G_{3(u)}=\mathrm{P}\left(X_{1}>u, X_{2}>u, X_{3} \leq u\right),
\end{aligned}
$$

and

$$
\begin{aligned}
& G_{12(u)}=\mathrm{P}\left(X_{1} \leq u, X_{2} \leq u, X_{3}>u\right), \\
& G_{13(u)}=\mathrm{P}\left(X_{1} \leq u, X_{2}>u, X_{3} \leq u\right), \\
& G_{23(u)}=\mathrm{P}\left(X_{1}>u, X_{2} \leq u, X_{3} \leq u\right) .
\end{aligned}
$$

We shall consider two new models. First, consider the collapsed symmetry $\left(C^{3}\right)$ model defined by, for $u=1, \ldots, r-1$,

$$
G_{1(u)}=G_{2(u)}=G_{3(u)},
$$

and

$$
G_{12(u)}=G_{13(u)}=G_{23(u)} .
$$

Consider the $r-1$ ways of collapsing the $r \times r \times r$ table into a $2 \times 2 \times 2$ table by choosing cut points after the category $u$ of $X_{1}, X_{2}$ and $X_{3}$ for $u=1, \ldots, r-1$ (see Table 2). Denote the cumulative probability for the $(i, j, k)$-th cell of the $u$-th collapsed $2 \times 2 \times 2$ table $(u=1, \ldots, r-1)$ by $G_{i j k(u)}^{*}(i=1,2 ; j=1,2 ; k=1,2)$. For instance, $G_{121(u)}^{*}=G_{13(u)}$ and $G_{212(u)}^{*}=G_{2(u)}$. Then the $C^{3}$ model is expressed as, for $u=1, \ldots, r-1$,

and

$$
G_{122(u)}^{*}=G_{212(u)}^{*}=G_{221(u)}^{*},
$$

$$
G_{112(u)}^{*}=G_{121(u)}^{*}=G_{211(u)}^{*} .
$$

Namely the $C^{3}$ model indicates the symmetry for the $u$-th collapsed $2 \times 2 \times 2$ table $(u=1, \ldots, r-1)$. Note that the $C^{3}$ model is not equivalent to the $S^{3}$ model in the $r \times r \times r$ table. Thus the $C^{3}$ model states that for each cut point $u$ $(u=1, \ldots, r-1),(1)$ from (2.1), three cumulative probabilities that one of $X_{1}$, $X_{2}$ and $X_{3}$ is in the category $u$ or below and the other two are in the category 
Table 2. Illustration of (a) the $3 \times 3 \times 3$ table of probabilities, and (b) the collapsed $2 \times 2 \times 2$ tables.

(a) The probabilities

\begin{tabular}{|c|c|c|c|c|c|c|c|c|c|}
\hline \multirow[b]{3}{*}{$X_{1}$} & & \multicolumn{7}{|c|}{$X_{3}$} & \\
\hline & & $X_{2}$ & & & $X_{2}$ & & & $X_{2}$ & \\
\hline & (1) & (2) & $(3)$ & (1) & (2) & $(3)$ & (1) & $(2)$ & (3) \\
\hline (1) & $p_{111}$ & $p_{121}$ & $p_{131}$ & $p_{112}$ & $p_{122}$ & $p_{132}$ & $p_{113}$ & $p_{123}$ & $p_{133}$ \\
\hline (2) & $p_{211}$ & $p_{221}$ & $p_{231}$ & $p_{212}$ & $p_{222}$ & $p_{232}$ & $p_{213}$ & $p_{223}$ & $p_{233}$ \\
\hline (3) & $p_{311}$ & $p_{321}$ & $p_{331}$ & $p_{312}$ & $p_{322}$ & $p_{332}$ & $p_{313}$ & $p_{323}$ & $p_{333}$ \\
\hline
\end{tabular}

(b) The $u$-th $(u=1,2)$ collapsed $2 \times 2 \times 2$ table

\begin{tabular}{|c|c|c|}
\hline$X_{3}$ & & \\
\hline \multicolumn{3}{|c|}{$X_{2}$} \\
\hline$X_{1}$ & $\leq u$ & $>u$ \\
\hline$\leq u$ & $\sum_{a=1}^{u} \sum_{b=1}^{u} \sum_{c=1}^{u} p_{a b c}\left(=G_{111(u)}^{*}\right)$ & $\sum_{a=1}^{u} \sum_{b=u+1}^{3} \sum_{c=1}^{u} p_{a b c}\left(=G_{13(u)}=G_{121(u)}^{*}\right)$ \\
\hline$>u$ & $\sum_{a=u+1}^{3} \sum_{b=1}^{u} \sum_{c=1}^{u} p_{a b c}\left(=G_{23(u)}=G_{211(u)}^{*}\right)$ & $\sum_{a=u+1}^{3} \sum_{b=u+1}^{3} \sum_{c=1}^{u} p_{a b c}\left(=G_{3(u)}=G_{221(u)}^{*}\right)$ \\
\hline$X_{3}$ & \multicolumn{2}{|c|}{$>u$} \\
\hline \multicolumn{3}{|c|}{$X_{2}$} \\
\hline$X_{1}$ & $\leq u$ & $>u$ \\
\hline$\leq u$ & $\sum_{a=1}^{u} \sum_{b=1}^{u} \sum_{c=u+1}^{3} p_{a b c}\left(=G_{12(u)}=G_{112(u)}^{*}\right)$ & $\sum_{a=1}^{u} \sum_{b=u+1}^{3} \sum_{c=u+1}^{3} p_{a b c}\left(=G_{1(u)}=G_{122(u)}^{*}\right)$ \\
\hline$>u$ & $\sum_{a=u+1}^{3} \sum_{b=1}^{u} \sum_{c=u+1}^{3} p_{a b c}\left(=G_{2(u)}=G_{212(u)}^{*}\right)$ & $\sum_{a=u+1}^{3} \sum_{b=u+1}^{3} \sum_{c=u+1}^{3} p_{a b c}\left(=G_{222(u)}^{*}\right)$ \\
\hline
\end{tabular}

$u+1$ or above, are the same, and (2) from (2.2), three cumulative probabilities that two of $X_{1}, X_{2}$ and $X_{3}$ are in the category $u$ or below and the other one is in the category $u+1$ or above, are the same. So, the $C^{3}$ model indicates the symmetry for $r-1$ collapsed $2 \times 2 \times 2$ tables.

We note that the $S^{3}$ model implies the $C^{3}$ model, and the $C^{3}$ model implies the $M^{3}$ model. Namely, the $C^{3}$ model is an extension of the $S^{3}$ model and it is a special case of the $M^{3}$ model (though only when $T=2$ the $C^{2}$ model is equivalent to the $M^{2}$ model).

Next consider the collapsed quasi-symmetry $\left(C Q S^{3}\right)$ model defined by, for $u=1, \ldots, r-1$,

$$
G_{i j k(u)}^{*}=\mu^{(u)} \alpha_{1(i)}^{(u)} \alpha_{2(j)}^{(u)} \alpha_{3(k)}^{(u)} \Psi_{i j k}^{(u)} \quad(i=1,2 ; j=1,2 ; k=1,2),
$$

where

$$
\Psi_{i j k}^{(u)}=\Psi_{j i k}^{(u)}=\Psi_{i k j}^{(u)}=\Psi_{j k i}^{(u)}=\Psi_{k i j}^{(u)}=\Psi_{k j i}^{(u)} .
$$

Note that without loss of generality, we may set, e.g., $\alpha_{1(2)}^{(u)}=\alpha_{2(2)}^{(u)}=\alpha_{3(2)}^{(u)}=1$ 
and $\Psi_{122}^{(u)}=\Psi_{222}^{(u)}=1$. A special case of this model obtained by putting $\alpha_{1(i)}^{(u)}=$ $\alpha_{2(i)}^{(u)}=\alpha_{3(i)}^{(u)}(u=1, \ldots, r-1 ; i=1,2)$ is the $C^{3}$ model.

Denote odds ratios as, for $u=1, \ldots, r-1$,

$$
\theta_{1}^{(u)}=\frac{G_{211(u)}^{*} G_{222(u)}^{*}}{G_{212(u)}^{*} G_{221(u)}^{*}}, \quad \theta_{2}^{(u)}=\frac{G_{121(u)}^{*} G_{222(u)}^{*}}{G_{122(u)}^{*} G_{221(u)}^{*}}, \quad \theta_{3}^{(u)}=\frac{G_{112(u)}^{*} G_{222(u)}^{*}}{G_{122(u)}^{*} G_{212(u)}^{*}} .
$$

Although the details are omitted, then the $C Q S^{3}$ model may be expressed, e.g., as

$$
\theta_{1}^{(u)}=\theta_{2}^{(u)}=\theta_{3}^{(u)} \quad(u=1, \ldots, r-1) .
$$

Namely, this indicates that for each of the $r-1$ collapsed $2 \times 2 \times 2$ tables, there is a structure of symmetry of odds ratios.

In the similar way to the decomposition of the symmetry model for the cell probabilities described in Section 1, we can obtain the following theorem:

THEOREM 1. The $C^{3}$ model holds if and only if both the $C Q S^{3}$ and $M^{3}$ models hold.

\subsection{Extension to multi-way tables}

Consider an $r^{T}(T>3)$ contingency table with ordered categories. Let $\left(j_{1}, \ldots, j_{k}, j_{k+1}, \ldots, j_{T}\right)$ be any permutation of $(1, \ldots, T)$. Then let for $u=$ $1, \ldots, r-1$, and $k=1, \ldots, T-1$,

$$
G_{j_{1} \ldots j_{k}(u)}=\mathrm{P}\left(X_{j_{1}} \leq u, \ldots, X_{j_{k}} \leq u, X_{j_{k+1}}>u, \ldots, X_{j_{T}}>u\right) .
$$

For instance, when $T=5$,

$$
\begin{aligned}
& G_{14(u)}=\mathrm{P}\left(X_{1} \leq u, X_{2}>u, X_{3}>u, X_{4} \leq u, X_{5}>u\right) \\
& G_{125(u)}=\mathrm{P}\left(X_{1} \leq u, X_{2} \leq u, X_{3}>u, X_{4}>u, X_{5} \leq u\right)
\end{aligned}
$$

For multi-way $r^{T}$ tables, consider the collapsed symmetry $\left(C^{T}\right)$ model defined by, for $u=1, \ldots, r-1$, and $k=1, \ldots, T-1$,

$$
G_{s_{1} \ldots s_{k}(u)}=\Delta_{k(u)} \quad\left(s_{j}=1, \ldots, T ; j=1, \ldots, k ; s_{l} \neq s_{m}(l \neq m)\right),
$$

where $\Delta_{k(u)}$ is unspecified.

Denote the cumulative probability for the $\left(i_{1}, \ldots, i_{T}\right)$-th cell of the $u$-th collapsed $2^{T}$ table $(u=1, \ldots, r-1)$ by $G_{i_{1} \ldots i_{T}(u)}^{*}$ where $i_{k}=1,2 ; k=1, \ldots, T$. Then the $C^{T}$ model may be expressed as, for $u=1, \ldots, r-1$,

$$
G_{i_{1} \ldots i_{T}(u)}^{*}=G_{j_{1} \ldots j_{T}(u)}^{*}
$$

where $\left(j_{1}, \ldots, j_{T}\right)$ is any permutation of $\left(i_{1}, \ldots, i_{T}\right)$ with $i_{k}=1,2 ; k=1, \ldots, T$. Namely, this model indicates the structure of symmetry for the $r-1$ ways of collapsing the $r^{T}$ table into a $2^{T}$ table. We note that the $C^{T}$ model is an extension of the $S^{T}$ model and it is a special case of the $M^{T}$ model. 
Next consider the collapsed quasi-symmetry $\left(C Q S^{T}\right)$ model defined by, for $u=1, \ldots, r-1$,

$$
G_{i_{1} \ldots i_{T}(u)}^{*}=\mu^{(u)} \alpha_{1\left(i_{1}\right)}^{(u)} \alpha_{2\left(i_{2}\right)}^{(u)} \cdots \alpha_{T\left(i_{T}\right)}^{(u)} \Psi_{i_{1} \ldots i_{T}}^{(u)},
$$

where $\Psi_{i_{1} \ldots i_{T}}^{(u)}=\Psi_{j_{1} \ldots j_{T}}^{(u)}$ and $\left(j_{1}, \ldots, j_{T}\right)$ is any permutation of $\left(i_{1}, \ldots, i_{T}\right)$ with $i_{k}=1,2 ; k=1, \ldots, T$. Note that without loss of generality, we may set, e.g., $\alpha_{1(2)}^{(u)}=\cdots=\alpha_{T(2)}^{(u)}=1$ and $\Psi_{12 \cdots 2}^{(u)}=\Psi_{22 \cdots 2}^{(u)}=1$. A special case of this model obtained by putting $\alpha_{1(i)}^{(u)}=\cdots=\alpha_{T(i)}^{(u)}(u=1, \ldots, r-1 ; i=1,2)$ is the $C^{T}$ model. Note that the $C Q S^{T}$ model can be defined when $T \geq 3$, and this model is different from the quasi-symmetry model with the multiplicative form for the cell probabilities described in Section 1.

In the similar way to the decomposition of the symmetry model for the cell probabilities for multi-way tables described in Section 1, we can obtain the following theorem:

THEOREM 2. The $C^{T}$ model holds if and only if both the $C Q S^{T}$ and $M^{T}$ models hold.

Note that each of the $C^{T}$ and $C Q S^{T}$ models is not invariant under the arbitrary same permutations of the categories for all variables, and thus each model should be applied for analyzing the multi-way tables with ordered categories.

\subsection{Goodness-of-fit test}

Let $n_{i_{1} \ldots i_{T}}$ denote the observed frequency in the $\left(i_{1}, \ldots, i_{T}\right)$-th cell of the $r^{T}(T \geq 2)$ table $\left(i_{k}=1, \ldots, r ; k=1, \ldots, T\right)$ with $n=\sum \ldots \sum n_{i_{1} \ldots i_{T}}$. Assume that $\left\{n_{i_{1} \ldots i_{T}}\right\}$ have a multinomial distribution. The maximum likelihood estimates of expected frequencies under each of the $C^{T}$ and $C Q S^{T}$ models could be obtained using the Newton-Raphson method in the log-likelihood equation.

Each model can be tested for goodness-of-fit by, e.g., the likelihood ratio chi-squared statistic (denoted by $G^{2}$ ) with the corresponding degrees of freedom. The numbers of degrees of freedom for each model are given in Table 3. Note that the number of degrees of freedom for the $C^{T}$ model is equal to the sum of those for the $C Q S^{T}$ and $M^{T}$ models.

Table 3. The numbers of degrees of freedom for models applied to the $r^{T}$ table $(T \geq 2)$, where the $C Q S^{T}$ model is defined when $T \geq 3$.

\begin{tabular}{cc}
\hline Models & Degrees of freedom \\
\hline$S^{T}$ & $r^{T}-K$ \\
$Q S^{T}$ & $r^{T}-K-(r-1)(T-1)$ \\
$C^{T}$ & $(r-1)\left(2^{T}-T-1\right)$ \\
$C Q S^{T}$ & $(r-1)\left(2^{T}-2 T\right)$ \\
$M^{T}$ & $(r-1)(T-1)$ \\
\hline Note: $K=\left(\begin{array}{c}r+T-1 \\
T\end{array}\right)=\frac{(r+T-1) !}{(r-1) ! T !}$.
\end{tabular}




\section{An example}

Consider the data in Table 4(a) obtained from the Meteorological Agency in Japan. These are obtained from the daily temperatures at Nagasaki City, Japan, in three years, 2001, 2002 and 2003, using three levels, (1) below normals, (2) normals and (3) above normals. The observation $n_{i j k}$ in the $(i, j, k)$-th cell in Table 4 (a) $(i=1,2,3 ; j=1,2,3 ; k=1,2,3)$ with $\Sigma \Sigma \Sigma n_{i j k}=365$ indicates that for each of $n_{i j k}$ days in 365 days (i.e., from 1 January to 31 December), the temperatures in three years are $i$ in 2001, $j$ in 2002, and $k$ in 2003. Table 4(b) gives two collapsed $2 \times 2 \times 2$ tables.

Table 5 gives the values of likelihood ratio statistics $G^{2}$ for testing goodnessof-fit of each model. The $S^{3}$ model fits these data poorly yielding $G^{2}=31.85$ with 17 degrees of freedom. The $C^{3}$ model fits these data well yielding $G^{2}=8.05$ with 8 degrees of freedom.

The $Q S^{3}$ model fits these data poorly, however, the $C Q S^{3}$ model fits the data well (see Table 5). In addition, we test that the $C^{3}$ model holds assuming that the $C Q S^{3}$ model holds true. According to the test based on the difference between the $G^{2}$ values for the $C^{3}$ and $C Q S^{3}$ models, the $C^{3}$ model is preferable to the $C Q S^{3}$ model.

The $M^{3}$ model also fits these data well (see Table 5). In addition, we test that the $C^{3}$ model holds assuming that the $M^{3}$ model holds true. According

Table 4. The daily temperatures at Nagasaki City, Japan, in 2001, 2002 and 2003. (The upper and lower parenthesized values are the maximum likelihood estimates of expected frequencies under the $C^{3}$ and $C Q S^{3}$ models, respectively.)

\begin{tabular}{|c|c|c|c|c|c|c|c|c|c|}
\hline \multirow[b]{3}{*}{2001} & \multicolumn{3}{|c|}{ (1) } & \multicolumn{3}{|c|}{$\begin{array}{l}2003 \\
(2)\end{array}$} & \multicolumn{3}{|c|}{ (3) } \\
\hline & \multicolumn{3}{|c|}{2002} & \multicolumn{3}{|c|}{2002} & \multicolumn{3}{|c|}{2002} \\
\hline & (1) & (2) & (3) & (1) & $(2)$ & (3) & (1) & $(2)$ & (3) \\
\hline \multirow[t]{3}{*}{ (1) } & 1 & 3 & 5 & 5 & 5 & 15 & 5 & 10 & 10 \\
\hline & $(1.00)$ & $(3.79)$ & $(5.86)$ & $(4.52)$ & $(5.56)$ & (15.56) & (5.13) & (13.02) & $(8.50)$ \\
\hline & $(1.00)$ & $(3.16)$ & $(5.82)$ & $(4.77)$ & $(5.00)$ & $(16.50)$ & $(4.84)$ & (10.13) & $(8.02)$ \\
\hline \multirow[t]{3}{*}{ (2) } & 5 & 18 & 9 & 15 & 41 & 31 & 18 & 20 & 24 \\
\hline & $(4.53)$ & $(18.55)$ & $(8.71)$ & $(13.28)$ & (41.00) & $(29.15)$ & (18.07) & $(23.06)$ & (18.81) \\
\hline & $(5.00)$ & $(17.81)$ & (9.78) & (15.15) & (41.00) & $(34.09)$ & $(18.43)$ & $(20.27)$ & $(19.25)$ \\
\hline \multirow[t]{3}{*}{ (3) } & 6 & 8 & 8 & 12 & 39 & 21 & 1 & 17 & 13 \\
\hline & $(5.12)$ & $(7.69)$ & (7.69) & (10.01) & $(36.46)$ & $(19.62)$ & $(1.28)$ & (26.03) & (13.00) \\
\hline & $(5.38)$ & $(7.11)$ & (8.11) & $(10.86)$ & (34.97) & $(21.52)$ & (1.35) & $(22.67)$ & (13.00) \\
\hline
\end{tabular}

(b) The $u$-th $(u=1,2)$ collapsed $2 \times 2 \times 2$ table $(u=1)$

\begin{tabular}{ccccc}
\hline & \multicolumn{2}{c}{$\leq 003$} & \multicolumn{2}{c}{$>u$} \\
\cline { 2 - 5 } 2001 & \multicolumn{2}{c}{2002} & \multicolumn{2}{c}{2002} \\
$\leq u$ & 1 & 8 & 10 & $>u$ \\
\hline & $(1.00)$ & $(9.65)$ & $(9.65)$ & $(42.64)$ \\
& $(1.00)$ & $(8.99)$ & $(9.61)$ & $(39.65)$ \\
$>u$ & 11 & 43 & 46 & 206 \\
& $(9.65)$ & $(42.64)$ & $(42.64)$ & $(207.13)$ \\
& $(10.38)$ & $(42.81)$ & $(45.79)$ & $(206.78)$ \\
\hline
\end{tabular}

\begin{tabular}{|c|c|c|c|c|}
\hline \multirow[b]{4}{*}{2001} & \multicolumn{4}{|c|}{2003} \\
\hline & \multicolumn{2}{|c|}{$\leq u$} & \multicolumn{2}{|c|}{$>u$} \\
\hline & \multicolumn{2}{|c|}{2002} & \multicolumn{2}{|c|}{2002} \\
\hline & $\leq u$ & $>u$ & $\leq u$ & $>u$ \\
\hline \multirow[t]{3}{*}{$\leq u$} & 93 & 60 & 53 & 34 \\
\hline & (92.23) & $(59.28)$ & $(59.28)$ & (27.31) \\
\hline & (92.90) & $(66.20)$ & $(53.67)$ & $(27.27)$ \\
\hline \multirow[t]{3}{*}{$>u$} & 65 & 29 & 18 & 13 \\
\hline & $(59.28)$ & $(27.31)$ & (27.31) & (13.00) \\
\hline & $(58.31)$ & (29.63) & $(24.02)$ & (13.00) \\
\hline
\end{tabular}


Table 5. Likelihood ratio chi-square values $G^{2}$ for models applied to the data in Table 4(a).

\begin{tabular}{ccc}
\hline Models & Degrees of freedom & $G^{2}$ \\
\hline$S^{3}$ & 17 & $31.85^{*}$ \\
$Q S^{3}$ & 13 & $28.14^{*}$ \\
$C^{3}$ & 8 & 8.05 \\
$C Q S^{3}$ & 4 & 4.61 \\
$M^{3}$ & 4 & 4.01 \\
\hline
\end{tabular}

* means significant at the 0.05 level.

to the test based on the difference between the $G^{2}$ values for the $C^{3}$ and $M^{3}$ models, the $C^{3}$ model is preferable to the $M^{3}$ model.

Since the $S^{3}$ model fits these data poorly, there is not a structure of complete symmetry for these data. However, since the $C^{3}$ model fits these data well, we can state that under the $C^{3}$ model, for $u(u=1,2)$, (1) three cumulative probabilities (i.e., $G_{1(u)}, G_{2(u)}$ and $\left.G_{3(u)}\right)$ that the temperature is in the level $u$ or below for one of three years, 2001, 2002 and 2003, and in the level $u+1$ or above for the other two years, are the same (without depending on in which year the temperature is lower than in the other two years), and (2) three cumulative probabilities (i.e., $G_{12(u)}, G_{13(u)}$ and $\left.G_{23(u)}\right)$ that the temperature is in the level $u$ or below for two of three years and in the level $u+1$ or above for the other one year, are the same (without depending on in which year the temperature is higher than in the other two years). Namely, there is no change of temperatures among 2001, 2002 and 2003 , in the sense that the symmetry of cumulative probabilities holds in two collapsed $2 \times 2 \times 2$ tables.

\section{Concluding remarks}

For the analysis of multi-way tables with nominal categories (not ordered categories), it is appropriate to apply the $S^{T}, Q S^{T}$ and $M^{T}$ models. Also, these models may be used for analyzing multi-way tables with ordered categories if we do not use the information of ordering of categories. We are also interested in models which can be used for analyzing multi-way tables with ordered categories. The $C^{T}$ and $C Q S^{T}$ models are not invariant under the arbitrarily same permutations of the categories for all variables. Thus, these models are suitable for the analysis of multi-way tables with ordered categories. Also these models are suitable when we want to see whether there is the structure of symmetry or quasi-symmetry for each of $r-1$ collapsed $2^{T}$ tables.

\section{Acknowledgements}

The authors would like to thank the referees for the helpful comments.

\section{REFERENCES}

Agresti, A. (1984). Analysis of Ordinal Categorical Data, Wiley, New York.

Agresti, A. (2002). Categorical Data Analysis, 2nd ed., Wiley, New York. 
Bhapkar, V. P. and Darroch, J. N. (1990). Marginal symmetry and quasi symmetry of general order, Journal of Multivariate Analysis, 34, 173-184.

Bishop, Y. M. M., Fienberg, S. E. and Holland, P. W. (1975). Discrete Multivariate Analysis: Theory and Practice, The MIT Press, Cambridge, Massachusetts.

Bowker, A. H. (1948). A test for symmetry in contingency tables, Journal of the American Statistical Association, 43, 572-574.

Caussinus, H. (1965). Contribution à l'analyse statistique des tableaux de corrélation, Annales de la Faculté des Sciences de l'Université de Toulouse, 29, 77-182.

McCullagh, P. (1978). A class of parametric models for the analysis of square contingency tables with ordered categories, Biometrika, 65, 413-418.

Miyamoto, N., Ohtsuka, W. and Tomizawa, S. (2004). Linear diagonals-parameter symmetry and quasi-symmetry models for cumulative probabilities in square contingency tables with ordered categories, Biometrical Journal, 46, 664-674.

Stuart, A. (1955). A test for homogeneity of the marginal distributions in a two-way classification, Biometrika, 42, 412-416.

Tomizawa, S. (1993). Diagonals-parameter symmetry model for cumulative probabilities in square contingency tables with ordered categories, Biometrics, 49, 883-887.

Tomizawa, S. (1995). A generalization of the marginal homogeneity model for square contingency tables with ordered categories, Journal of Educational and Behavioral Statistics, 20, 349-360.

Tomizawa, S. and Miyamoto, N. (2007). Diagonal uniform association symmetry models for cumulative probabilities in square tables, Advances in Statistical Analysis, 91, 269-278.

Tomizawa, S. and Tahata, K. (2007). The analysis of symmetry and asymmetry: Orthogonality of decomposition of symmetry into quasi-symmetry and marginal symmetry for multi-way tables, Journal de la Société Francaise de Statistique, 148, 3-36.

Tomizawa, S., Miyamoto, N. and Hatanaka, Y. (2001). Measure of asymmetry for square contingency tables having ordered categories, Australian and New Zealand Journal of Statistics, 43, 335-349.

Tomizawa, S., Miyamoto, N. and Yamamoto, K. (2006). Decomposition for polynomial cumulative symmetry model in square contingency tables with ordered categories, Metron, 64, 303-314.

Tomizawa, S., Miyamoto, N., Yamamoto, K. and Sugiyama, A. (2007). Extensions of linear diagonals-parameter symmetry and quasi-symmetry models for cumulative probabilities in square contingency tables, Statistica Neerlandica, 61, 273-283.

Yamamoto, H., Iwashita, T. and Tomizawa, S. (2007). Decomposition of symmetry into ordinal quasi-symmetry and marginal equimoment for multi-way tables, Austrian Journal of Statistics, 36, 291-306. 